\title{
Surgical Techniques for Dealing with Intraluminal Thrombus of Great Vessels in Advanced Differentiated Thyroid Carcinoma
}

\author{
${ }^{1}$ Mallika Dhanda, ${ }^{2}$ Akshay Anand, ${ }^{3}$ Devenraj Vijayant, ${ }^{4}$ Abhinav A Sonkar, ${ }^{5}$ Kul R Singh, ${ }^{6}$ Pooja Ramakant, ${ }^{7}$ Anand Mishra
}

\section{ABSTRACT}

Aim: We aim to define and refine the surgical technique for dealing with intraluminal thrombus of great vessels in advanced differentiated thyroid carcinoma (DTC) to reduce the morbidity.

Background: Venous tumor thrombus from DTC is a rare occurrence with sequelae that cause increased morbidity and early mortality. Management of such patients poses a challenge to surgeons.

Materials and methods: We define the surgical planning and road map for surgical management of advanced DTC with tumor thrombus involving the internal jugular vein (IJV) and superior vena cava (SVC) by sacrificing one IJV and retrieving the thrombus from the SVC by Fogarty catheter. This technique has minimal morbidity with good outcome.

Conclusion: By appropriate planning and meticulous surgical techniques, we can aggressively manage patients of advanced DTC with venous tumor thrombus and reduce the morbidity.

Clinical significance: By surgical techniques leading to complete surgical resections and saving native vital structures, we can aim for successful aggressive surgical management of advanced DTC with venous tumor thrombus.

Keywords: Differentiated thyroid cancer, Thyroid, Venous thrombus.

How to cite this article: Dhanda $M$, Anand $A$, Vijayant $D$, Sonkar AA, Singh KR, Ramakant P, Mishra A. Surgical Techniques for Dealing with Intraluminal Thrombus of Great Vessels in Advanced Differentiated Thyroid Carcinoma. World J Endoc Surg 2017;9(3):115-117.

Source of support: Nil

Conflict of interest: None

\footnotetext{
${ }^{1}$ Resident, ${ }^{2,5}$ Assistant Professor, ${ }^{3,6}$ Associate Professor ${ }^{4,7}$ Professor

1,2,4 Department of General Surgery, King George's Medical University, Lucknow, Uttar Pradesh, India

${ }^{3}$ Department of Cardio Thoracic and Vascular Surgery, King George's Medical University, Lucknow, Uttar Pradesh, India

${ }^{5-7}$ Department of Endocrine and Breast Surgery, King George's Medical University, Lucknow, Uttar Pradesh, India

Corresponding Author: Kul R Singh, Assistant Professor Department of Endocrine and Breast Surgery, King George's Medical University, Lucknow, Uttar Pradesh, India, Phone: +919795343700, e-mail: kulranjan@hotmail.com
}

\section{INTRODUCTION}

Venous tumor thrombus of differentiated thyroid cancer is a rare occurrence, and extension of tumor thrombus into SVC even rarer. Management of such cases remains a challenge. We aim to describe the management of a patient with follicular thyroid cancer with SVC thrombus and isolated clavicular metastasis and describe the detailed surgical techniques for surgical extirpation of tumor thrombus. A 68-year-old lady (Fig. 1) presented with a slowly progressing swelling in lower aspect of neck of 15 years duration along with a rapidly progressing swelling in the right clavicle and exertional dyspnea. Fine needle aspiration cytology from the thyroid swelling was suggestive of papillary thyroid carcinoma. A Tru-Cut biopsy from the thyroid swelling and clavicular swelling was suggestive of metastatic follicular thyroid carcinoma. Contrast-enhanced computed tomography (CECT) was suggestive of a $7.5 \times 5.5 \mathrm{~cm}$ well-defined swelling arising from the right lobe of thyroid. Another $11 \times 7.5 \times 6 \mathrm{~cm}$ osteolytic mass involving the medial end of the right clavicle compressing the right carotid and right subclavian vessels. The CECT also showed a thrombus extending from the middle portion of IJV to the terminal portion of SVC.

She was planned for a surgery followed by adjuvant whole body radioactive iodine therapy.

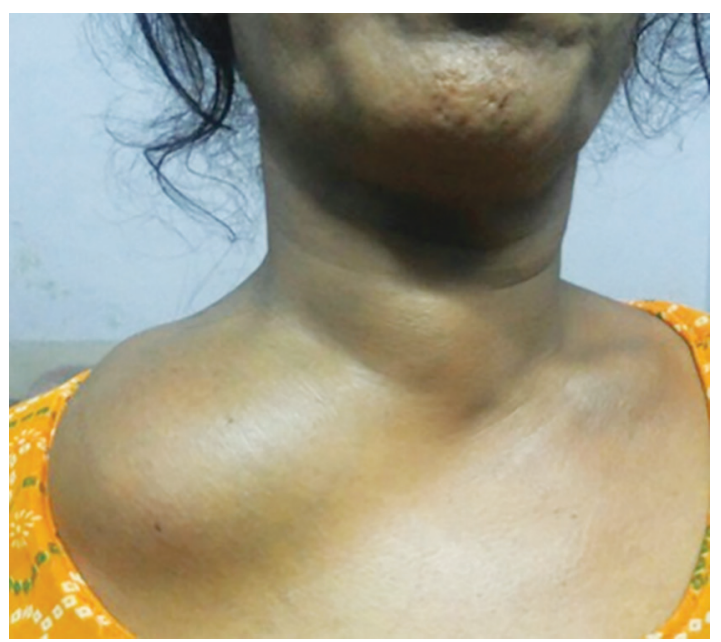

Fig. 1: Preoperative view of patient with right solitary thyroid nodule and right clavicular mass 


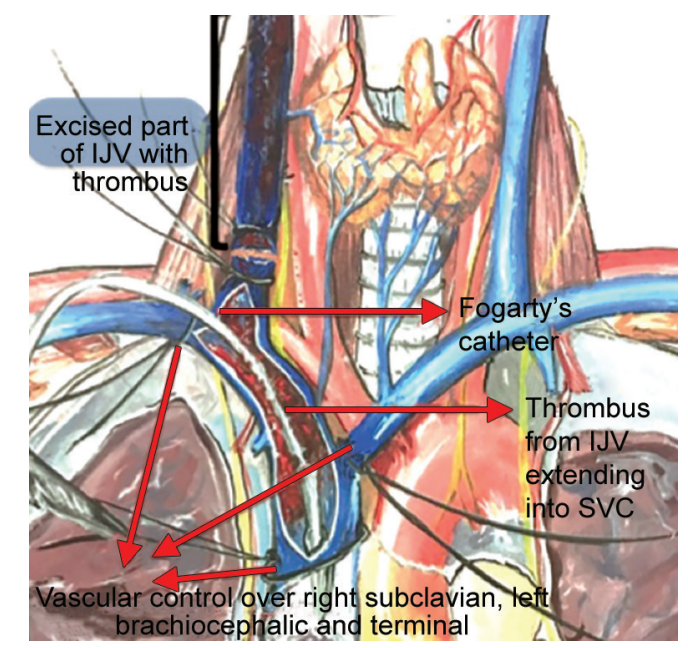

Fig. 2: Illustrative view of vascular controls and SVC thrombectomy

\section{MATERIALS AND METHODS}

We intend to describe the surgical technique of SVC tumor thrombectomy along with Total Thyroidectomy and clavicular metastasectomy. Under general anesthesia and thyroid position, subplatysmal flaps were raised following a large Kocher's incision. An upfront median sternotomy was performed. The strap muscles were divided and a total thyroidectomy performed via capsular dissection after opening up the carotid sheath and lateralizing the great vessels in the neck. Bilateral recurrent laryngeal nerve and superior parathyroid glands were identified and preserved in situ. The pericardium was excised and vascular control of SVC with the help of vascular tapes taken to prevent any thrombus embolization (Fig. 2). Subsequent controls of right subclavian and innominate veins were taped (Fig. 2). The right IJV was clamped and excised between a point superior to the tumor thrombus and just proximal to it joining the right subclavian vessel. The vascular tapes secured in the SVC, left innominate, and right subclavian vein were tightened, and a 5 French Fogarty's catheter was inserted into the SVC through a purse-string controlled venotomy in the right brachiocephalic vein and tumor thrombi retrieved piecemeal, ensuring a free channel. The vascular tape between the terminal portion of SVC and right atrium was secured to prevent migration of thrombi during the manipulation of Fogarty's catheter and thrombus retrieval. The right IJV was then secured with prolene sutures at the point of transection. The medial end of the clavicle was excised and right neck dissection done followed by meticulous hemostasis and wound closure in layers with appropriate drains in situ. The preclosure photograph and excised specimen is shown in Figure 3. Following an uneventful recovery (Fig. 4), patient has been receiving periodical radioactive iodine for the last 2 years with foci of uptake in the mediastinum, but remained totally asymptomatic with elevated but reducing stimulated serum thyroglobulin values $(40 \mathrm{ng} / \mathrm{mL})$.

\section{DISCUSSION}

Venous thrombus extending from IJV to SVC in DTC is rare with significant morbidity and mortality, if not managed appropriately. ${ }^{1}$ The tumor extension sets off at the thyroid veins due to invasion of malignant cells followed by fibrin deposition and continued growth. The intraluminal extension should be differentiated from extraluminal vascular invasion as their management differs. ${ }^{2}$ A significant proportion of patients with venous thrombus are asymptomatic for the same at presentation. These are very likely to become symptomatic for the same in due course of time. ${ }^{3}$ Intraluminal extension is not an absolute contraindication to surgical management of these patients. Tumor thrombus does not necessarily invade
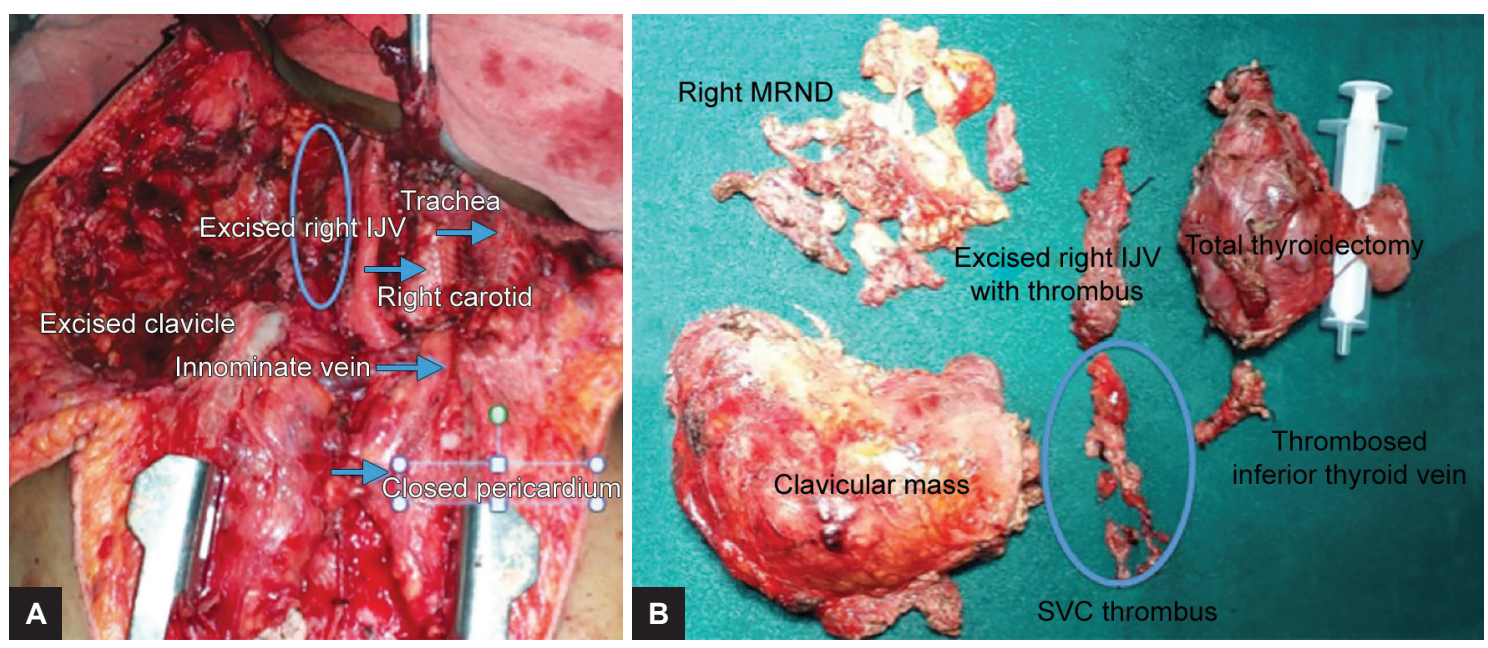

Figs 3A and B: Surgical site: (A) Preclosure photograph; and (B) excised specimen 


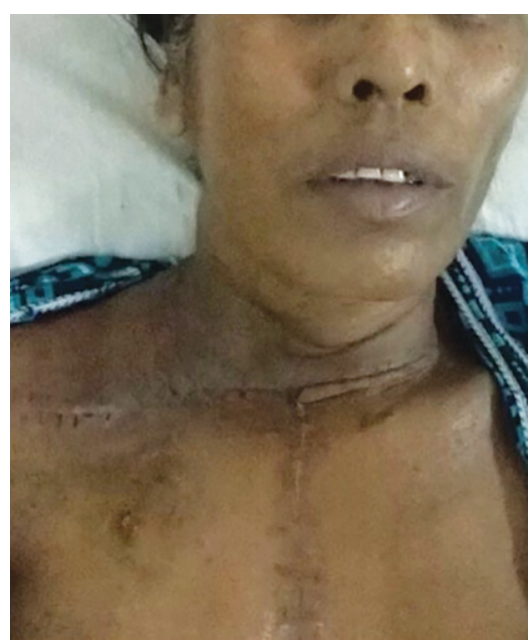

Fig. 4: Postoperative view of patient

the vascular wall in all cases. These cases have been successfully managed with tumor thrombectomy and occasionally by SVC grafts. ${ }^{4}$ The IJV can be safely transected and excised on one side without any adverse clinical consequences. A rim of contrast (ring sign) around the venous thrombus in CECT indicates that thrombectomy may be a feasible surgical procedure. ${ }^{5}$ Cardiopulmonary bypass is necessary in cases if the tumor thrombus has progressed to the right atrium. These interventions followed by adjuvant radioactive iodine treatment would delay, if not decrease, the incidence of potential sequela like SVC syndrome, tumor embolism, and fatal right atrial obstruction. The role of external beam radiotherapy in DTC remains controversial, but is very likely to benefit those with gross residual disease, and, occasionally, venous obstruction by thyroid cancer responds very well to same. ${ }^{1}$ These interventions are likely to increase the reported survival of such patients whose reported survival has ranged between 12 days and 5 years. ${ }^{6}$
It is important to understand the finer intricacies of dealing with patients with intraluminal thrombus to achieve best possible rates of cure or palliation.

\section{CONCLUSION}

We recommend aggressive management in DTC with intraluminal tumor thrombus of great vessels.

\section{ACKNOWLEDGMENT}

Authors would like to thank Dr Sanjeev Kumar, MBBS student, King George's Medical University, Lucknow, for his pictorial representation of intraoperative scenario.

\section{REFERENCES}

1. Hyer SL, Dandelkar P, Newbold K, Haq M, Wechalakar K, Thway K, Harmer C. Thyroid cancer causing obstruction to great veins in the neck. World J Surg Oncol 2008 Apr;6:36.

2. Dikici AS, Yildirim O, Er ME, Kilic F, Tutar O, Kantarci F, Mihmanli I. A rare complication of Thyroid malignancies: jugular vein invasion. Pol J Radiol 2015 Jul;80:360-363.

3. Marcy PY, Thariat J, Bozec A, Poissonnet G, Benisvy D, Dassonville $\mathrm{O}$. Venous obstruction of thyroid malignancy origin: the Antoine Lacasagne Institute experience. World J Surg Oncol 2009 Apr;7:40.

4. Wada N, Masudo K, Hirakawa S, Woo T, Arai H, Suganuma N, Iwaki H, Yukawa N, Uchida K, Imoto K, et al. Superior vena cava reconstruction using autologous tissue in two cases of differentiated thyroid carcinoma presenting with SVC syndrome. World J Surg Oncol 2009 Oct;7:75.

5. Franco IF, Gurrado A, Lissidini G, Di Meo G, Pasculli A, Testini M. Floating left Innominate vein neoplastic thrombus: a rare case of mediastinal extension of follicular thyroid carcinoma. Phebology 2015 Mar;30(2):140-144.

6. Gross M, Mintz Y, Maly B, Pinchas R, Muggia-Sullam M. Internal jugular vein tumor thrombus associated with thyroid carcinoma. Ann Otol Rhinol Laryngol 2004 Sep;113(9): 738-740. 